\title{
Bone marrow-derived cells are differentially involved in pathological and physiological retinal angiogenesis in mice.
}

\section{$\operatorname{AUTHOR}(\mathrm{S})$ :}

Zou, He; Otani, Atsushi; Oishi, Akio; Yodoi, Yuko; Kameda, Takanori; Kojima, Hiroshi; Yoshimura, Nagahisa

\section{CITATION:}

Zou, He ... [et al]. Bone marrow-derived cells are differentially involved in pathological and physiological retinal angiogenesis in mice.. Biochemical and biophysical research communications 2009, 391(2): 1268-1273

\section{ISSUE DATE:}

2009-12-17

URL:

http://hdl.handle.net/2433/92976

\section{RIGHT:}

c 2009 Elsevier Inc. All rights reserved.; この論文は出版社版でありませ ん。引用の際には出版社版をご確認ご利用ください。; This is not the published version. Please cite only the published version. 
Bone marrow-derived cells are differentially involved in pathological and physiological retinal angiogenesis in mice

He Zou MD.*; Atsushi Otani M.D., PhD; Akio Oishi M.D., PhD; Yuko Yodoi M.D.,

PhD; Takanori Kameda M.D., Hiroshi Kojima M.D., and Nagahisa Yoshimura M.D.,

$\mathrm{PhD}$

Department of Ophthalmology and Visual Sciences, Kyoto University Graduate School

of Medicine, Kyoto 606-8507, Japan

*present address: Department of Ophthalmology, The Second Hospital of Ji Lin

University, Chang Chun 130031, Ji Lin China

Correspondence to: Atsushi Otani

Department of Ophthalmology and Visual Sciences, Kyoto University Graduate School of Medicine, Shougoin Kawaharacho 54, Sakyouku, Kyoto 606-8507, Japan

Tel: (81)-75-751-3248; Fax: (81)-75-752-0933

E-mail address: otan@kuhp.kyoto-u.ac.jp 


\begin{abstract}
PURPOSE. Bone marrow-derived cells have been shown to play roles in angiogenesis. Although these cells have been shown to promote angiogenesis, it is not yet clear whether these cells affect all types of angiogenesis. This study investigated the involvement of bone marrow-derived cells in pathological and physiological angiogenesis in the murine retina.
\end{abstract}

MATERIALS AND Methods. The oxygen-induced retinopathy (OIR) model was used as a retinal angiogenesis model in newborn mice. To block the influence of bone marrowderived cells, the mice were irradiated with a 4-Gy dose of radiation from a ${ }^{137} \mathrm{Cs}$ source. Irradiation was performed in four different conditions with radio dense 2-cm thick lead disks ; (1) H group, the head were covered with these discs to protect the eyes from radiation; (2) A group, all of the body was covered with these discs; (3) N group, mice were completely unshielded; (4) C group, mice were put in the irradiator but were not irradiated. On P17, the retinal areas showing pathological and physiological retinal angiogenesis were measured and compared to the retinas of non-irradiated mice.

RESULTS. Although irradiation induced leukocyte depletion, it did not affect the number of other cell types or body weight. Retinal nonperfusion areas were significantly larger in irradiated mice than in control mice $(P<0.05)$, indicating that physiological angiogenesis was impaired. However, the formation of tuft-like angiogenesis processes was more prominent in the irradiated mice $(P<0.05)$, indicating that pathological angiogenesis was intact.

Conclusions. Bone marrow-derived cells seem to be differentially involved in the formation of physiological and pathological retinal vessels. Pathological angiogenesis in the murine retina does not require functional bone marrow-derived cells, but these cells 
are important for the formation of physiological vessels. Our results add a new insight into the pathology of retinal angiogenesis and bolster the hypothesis that bone marrow cells are involved in the pathology or severity of retinal angiogenic diseases.

Key words

Bone marrow derived stem cell, retinal angiogenesis, pathological angiogenesis, physiological angiogenesis 


\section{Introduction}

Angiogenesis is associated with retinal diseases such as diabetic retinopathy, retinopathy of prematurity and retinal vein occlusion. The common underlying pathological condition of those diseases is retinal hypoxia caused by a loss of functional vessels. The hypoxic retina releases angiogenic cytokines that are key players in retinal angiogenesis. Among these factors, vascular endothelial growth factor (VEGF) is key,[1, 2] and the efficacy of an anti-VEGF strategy has been demonstrated in clinical practice.

Although it is clear that cytokines play a central role in angiogenesis, it is also becoming evident that the mechanism of angiogenesis is more complicated than previously thought and that there are many factors involved in the process. In particular, the involvement of bone marrow (BM)-derived stem and progenitor cells has become a new paradigm in the mechanism of angiogenesis. Initially, the effect of BM-derived cells was reported to be mediated mainly by their differentiation into pericyte/smooth muscle cells [3-6] and/or endothelial cells [7, 8] that were incorporated into the walls of new vessels. Later studies suggested an alternative pathway in which the stem cells contributed to the neovascular process by their paracrine effects $[9,10]$. In fact, a variety of cytokines are released by stem cells in vitro, including MCP-1, VEGF-A, FGF-2, and IL-6. [10] All of these cytokines have been shown to have positive effects on promoting new vessel formation [10].

Angiogenesis can be physiological or pathological [11]. Physiological angiogenesis is exemplified by neovascularization in embryogenesis: In this process, physiological blood vessels are formed. In contrast, pathological angiogenesis is associated with disease conditions such as cancer, age-related macular degeneration, retinopathy of prematurity, and diabetic retinopathy: In these diseases, non- 
physiological vasculature develops. In terms of the retina, pathological angiogenesis is the major pathogenesis that causes functional impairment. Although there are some overlaps between the two types of angiogenesis, the underlying mechanisms have marked differences [11].

In the retina, the two kinds of angiogenesis can be distinguished by the location of the new blood vessels: Intraretinal angiogenesis is seen mostly in physiological angiogenesis during development, while extraretinal angiogenesis occurs on the surface of the retina. The latter is a type of pathological angiogenesis that causes severe vision loss in many ischemic retinal diseases. Although little is known about the mechanistic differences between intra- and extraretinal angiogenesis, induction of intraretinal angiogenesis rather than extraretinal angiogenesis in the ischemic retina has potential as a therapeutic strategy.

In the present study, we investigated whether BM-derived cells have different roles in intra- and extraretinal angiogenesis using the murine oxygen-induced retinopathy (OIR) model. The OIR mouse model is a hypoxia-induced retinal neovascularization model in which intraretinal and extraretinal angiogenesis develop simultaneously [12].

\section{Materials and Methods}

\section{The oxygen-induced retinopathy (OIR) model}

Mice were treated according to the regulations put forth in the ARVO "Statement for the Use of Animals in Ophthalmic and Vision Research" and the "Guidelines for Animal Experimentation” at Kyoto University. All animals were obtained from Japan SLC (Shizuoka, Japan) and housed in the Institute of Laboratory Animals at the 
Graduate School of Medicine of Kyoto University. OIR was induced in newborn C57BL/6 mice as described by Smith et al. [13] Briefly, on postnatal day 7 (P7), newborn mice were placed along with their dams into a $75 \%$ oxygen environment (Fig. 1). On P12, after 5 days in this environment, mice were transferred back to a normal laboratory environment (room air). During this period, the room temperature was maintained at $20^{\circ} \mathrm{C}$, and illumination was provided by standard fluorescent lighting on a 12-hour light-dark cycle. Pups were nursed by their dams, and dams were given food and water ad libitum.

\section{${ }^{137}$ Cs-gamma-photon irradiation}

Immediately after their return to room air on day P12, mice were irradiated with a Gammacell 40 Exactor (Nordion, Ottawa, Canada) to impair the function of BM cells (Fig. 1A). As shown in Figure 1B, irradiation was performed using radiodense 2-cm thick lead disks to protect the head $(\mathrm{H})$ or entire body (all-covered, A) of mice; alternatively, mice were completely unshielded (noncovered, N). The control group (C) mice were put in the irradiator but were not irradiated.

Five different doses of radiation, ranging from 2 to $6.5 \mathrm{~Gy}$, were tested to identify a dose that would affect leukocyte levels but not erythrocyte levels. Mice were irradiated on day P12, and hematological examination was performed on P17 (Erma Particle Counter PCE 170, Erma Inc, Tokyo, Japan). The mice were weighed 1, 3, and 5 days after irradiation to determine the effect of radiation, if any, on general development.

\section{Bone marrow transplantation in OIR model}


Lethal irradiation (7.5 Gy) was performed to the recipient mice (P12). Bone marrow is obtained by slowly flushing medium (RPMI 1640 culture medium containing $2.5 \%$ HEPES (1M) and 1\% gentamicin) inside the diaphyseal channel of femur and tibia of the donor mice (C57BL/6-Tg CAG-EGFP, SLC Inc., Shizuoka, Japan) with a syringe through a 27-gauge needle. The cells were injected to the irradiated recipient mice intraperitoneally ( $200 \mu \mathrm{L} /$ mouse). The retinas of recipient mice were examined at P26.

\section{Retinal whole-mount preparation and immunohistochemistry}

Retinal whole-mounts were prepared as described previously [14]. Briefly, mouse retinas were harvested and fixed with $4 \%$ paraformaldehyde and methanol, then blocked using $50 \%$ fetal bovine serum/20\% normal goat serum for 1 hour at room temperature before exposure to antibodies. The following primary antibodies were used: anti-CD31 (BD Biosciences Pharmingen, San Diego, CA) to stain vascular endothelial cell, anticollagen type IV (Chemicon, Temecula, CA) to stain vascular collagen IV, and antiGFAP (Dako, Tokyo Japan) for astrocyte staining. The secondary antibodies were Alexa 488- or 594-conjugated antibodies (Invitrogen, Eugene, OR).

\section{Imaging and quantifying the areas of nonperfusion and neovascular tufts}

Images of the retina were obtained with a Microscope Axio Imager (Carl Zeiss, Jena, Germany), and quantification was performed using Axiovision version 4.3 software (Carl Zeiss). Whole retinal montages were created by the software based on defined landmarks such as the optic disc and major vessels. Quantification of areas of nonperfusion and neovascular tufts was performed on these montages as described previously [15]. In short, areas of nonperfusion and neovascular tufts were selected 
using Photoshop (Adobe, Tokyo, Japan) and high-resolution images of immunostained retinal whole mounts (Fig. 3G and $3 \mathrm{H}$ ). The total areas were expressed in pixels that were then converted to square micrometers. The conversion factor was calculated to be $32.17 \mu \mathrm{m}^{2} /$ pixel for our set-up.

\section{Statistical analysis}

All results were presented as the mean \pm SD unless otherwise noted. Statistical analysis was performed using SPSS statistical software. Data were compared between groups using one-way ANOVA followed by the Bonferroni test or unpaired t-test as appropriate. The $P$ value $<0.05$ was considered statistically significant.

\section{Results}

\section{Effect of 4-Gy irradiation on murine development and hematological cell count}

Five different doses of radiation were tested to identify an optimal radiation dose that would reduce leukocyte levels but not erythrocyte levels. We found that a 4-Gy dose delivered using a $1.11 \mathrm{~Gy} /$ minute dose rate significantly impaired the leukocyte number but not the erythrocyte number. A dose greater than 5 Gy significantly reduced both leukocytes and erythrocytes. At a 4-Gy irradiation dose, the hemoglobin concentration was not affected significantly (Fig. 2A). The mean weight of mice exposed to 4-Gy radiation was not reduced compared to control animals on days 1 (P13), 3 (P15), or 5 (P17) after radiation (Fig. 2B). We concluded that a 4-Gy dose of radiation did not delay the normal development of these animals, at least during the experimental period.

The 4-Gy dose of irradiation on P12 did not significantly change either the leukocyte or erythrocyte count (determined on P17) in the A group (WBC: $2.0 \pm 0.3$ 
$\mathrm{x} 10^{3} /$ micro L, RBC: $5.9 \pm 0.2 \times 10^{6} /$ micro L) compared to the C group (WBC: $2.4 \pm 0.8$ $\mathrm{x} 10^{3} /$ micro L, RBC: $6.4 \pm 0.5 \times 10^{6} /$ micro L) (Fig. 2C). In contrast, 4-Gy irradiation to the body ( $\mathrm{H}$ group) significantly reduced the leukocyte count to $0.7 \pm 0.3 \times 10^{3} / \mathrm{micro} \mathrm{L}$; the erythrocyte count and hemoglobin concentration did not differ significantly in the $\mathrm{H}$ group compared to the $\mathrm{C}$ and $\mathrm{A}$ groups. In addition, the 4-Gy dose irradiation eliminated the colony forming unit (CFU-Hill) in the peripheral blood.

\section{Definition of physiological and pathological angiogenesis in the OIR model}

Retinal whole mounts were analyzed for OIR on P17 (n=9). Exposure to high levels of oxygen causes marked retinal capillary inhibition, especially in the central area (Fig. 3A). After the mice were transferred back to a normal environment (i.e. to room air), new vessels grew from the superficial plexus at the transition between the perfusion and nonperfusion areas; the vessels extended to the central avascular areas. As shown in Figure 3B and 3C, physiological (intraretinal) angiogenesis was observed as extending filopodias that colocalized with the underlying astrocytic processes. In addition, numerous neovascular tufts were observed in the mid-peripheral region of the retinas (Fig. 3B and 3E). Unlike physiological angiogenesis, which extended to the central avascular areas, the tufts were not associated with astrocytes (Fig. 3F) and were highly abnormal in appearance, consisting of swirls of capillaries folded in close proximity to each other and breaking through the inner limitans to the vitreous cavity. This type of angiogenesis is typical of pathological angiogenesis in the OIR model.

\section{Leukocyte depletion affects physiological angiogenesis but not pathological angiogenesis}


In the OIR model, the nonperfusion areas were largest on P12 in control mice (4301.0 \pm $505.9 \mathrm{~mm}^{2}$ ) and gradually decreased as physiological angiogenesis progressed (described above). The avascular areas, which represent physiological angiogenesis, and the areas with tufts, which represent pathological angiogenesis, were measured on P17 and compared (Fig. 4A-D).

There was no significant difference in the size of the avascular areas in A group mice and control mice (C: $1990 \pm 570.47$, A: $2190 \pm 389.89 \mathrm{~mm}^{2,} P<0.05$; Fig. 4E). In addition, the areas with neovascular tufts in the A group were not significantly different than controls (C: $385 \pm 64.38$, A: $416 \pm 70.78 \mathrm{~mm}^{2}$; Fig. 4F).

In contrast, in group $\mathrm{H}$, the 4-Gy dose of irradiation significantly increased both the mean area of nonperfusion (to $2660.0 \pm 349 \mathrm{~mm}^{2}, P<0.05$ ) and the mean area of neovascular tufts (to $500.0 \pm 96.8 \mathrm{~mm}^{2}, P<0.05$ ). In addition, in group $\mathrm{N}$, the mean avascular area and neovascular area were larger than that of the other groups (data not shown); however, we could not exclude the possibility that irradiation directly affected retinal angiogenesis.

\section{Bone marrow derived cells migrate into the avascular areas in OIR model}

To investigate whether bone marrow derived cells participate in the neovascularization of OIR model, bone marrow transplantation was performed with GFP+ bone marrow on P12, when the mice were transferred back to a normal laboratory environment (room air) after 5 days in $75 \% \mathrm{O}_{2}$ environment. The retina was examined at $\mathrm{P} 26$. Bone marrow derived cells (green) were observed especially in avascular area of the retina where physiological angiogenesis will develop and only a small number of cells were observed around the tufts. (Supplemental Fig. 1) 


\section{Discussion}

In the present study, we showed that depletion of BM-derived cells in the OIR model, including leukocytes and BM-derived stem cells, leads to an increase in the retinal avascular area and in neovascular tufts. In addition, we found that bone marrow derived cells seem to be mobilized more in the avascular area than the neovascular tufts in the model. These results suggest that physiological intraretinal angiogenesis is controlled by BM-derived stem cells, and that pathological extraretinal angiogenesis is not affected fully by these cells.

Irradiation to just part of the body, excluding the head, also affected the formation of physiological and pathologic angiogenesis in the mouse OIR model. The all-covered A group had almost the same mean avascular and neovascular area as the non-irradiated $\mathrm{C}$ group, indicating that the lead cover effectively shielded the mice from radiation. In contrast, the head-covered $\mathrm{H}$ group had significantly larger avascular and neovascular areas compared to the non-irradiated control (C) mice. These data showed that the decreased physiological angiogenesis and increased pathological angiogenesis was primarily the result of irradiation to the body rather than to the eye.

Considering that these changes were observed very early after irradiation, the depletion of BM-derived cells is the most likely cause of the decreased vascular formation. Although damage to the esophagus, gastrointestinal tract, and urinary organs can also occur right after irradiation, the development of the mice was almost normal, indicating that the damage to these systems had a minimal impact in the present study. Among the BM-derived cells, the WBC population seemed to contain the cells 
responsible for the observed effects because the number of RBCs was not significantly decreased even in whole-body irradiated mice.

Among the WBCs, circulating hematopoietic stem cells (HSCs) are good candidates for being responsible for the differences we observed in vascular formation in the present study. The cells are derived from bone marrow [16] and have been shown to participate in normal and pathological postnatal angiogenesis [17]. HSCs circulate and contribute to angiogenesis/vasculogenesis, as demonstrated in experimental models of myocardial infarction [18] and hind limb [19], corneal [5], and choroid development $[6,20]$. Several clinical studies have reported the efficacy of BM-derived cells in promoting angiogenesis in ischemic diseases [21-23]. Although BM-derived stem cells are considered a potential therapy with a strong angiogenic effect, their exact role in promoting angiogenesis is still unclear. BM-derived stem cells differentiate into mature endothelial cells, perivascular cells, and into other cell types that control angiogenesis directly or indirectly. It has even been suggested that BM-derived cells work as an antiangiogenic agent in some circumstances.

BM-derived cells may be involved both directly and indirectly in retinal and choroidal angiogenesis. Ritter et al. reported that BM-derived cells administered intravitreally promote physiological retinal angiogenesis [24]. Intravitreal injection of BM-derived progenitor cells promoted revascularization of nonperfusion area and reduced neovascular tuft formation in the OIR model. They hypothesized that neovascular tuft formation was reduced following physiological revascularization or that the injected BM cells acted as immune suppressive agents and reduced the output of tuft-inducing angiogenic factors. Ishida et al. reported that leukocytes adhere to vasculature and prune immature vessels via Fas-FasL-mediated selective apoptosis 
during development [25]. Taken together, these data suggest that BM-derived cells may have roles both in new vessel formation and in excessive vessel pruning in the retina.

Similarly, the role of BM-derived cells in choroidal neovascularization (CNV) remains unclear. BM-derived cells can participate in the formation of $\mathrm{CNV}{ }^{2}$ however, it is difficult to determine the effect of those cells in CNV formation because the cells might differentiate into many different cell types [26]. We have suggested that BMderived cells may act as CNV suppressors after investigating circulating BM-derived cells both in age-related macular degeneration (AMD) patient [27] and in vitro (presented at ARVO 2008).

Considering earlier data, it is possible that BM-derived cells might have a biphasic effect in angiogenesis that can be changed by environmental factors and angiogenesis conditions. In the present study, we developed a unique method to suppress BM-derived cells. Our results suggest that the development of physiological vessels and abnormal neovascular might be controlled by different mechanisms in the retina. Considering that neovascular tuft formation occurred after almost complete loss of leukocytes, most likely including BM-derived progenitor cells, the formation of pathological angiogenesis that is observed in diabetic retinopathy and retinopathy of prematurity might be independent of BM-derived cells. Of course, this result does not exclude the involvement of BM-derived cells in pathological angiogenesis, but it indicates that the involvement is not as strong as the involvement of BM-derived cells in physiological angiogenesis of the retina. The function of BM-derived stem cells has been reported to reduce markedly with age and with diseases such as diabetes. The results of this study explain the enlargement of the avascular area along with the development of extraretinal neovascularization that is observed in patients with 
advanced diabetes. We are currently investigating the correlation between the status of diabetic retinopathy and the function of BM-derived cells.

There were limitations to this study. First, we did not identify the particular cell types responsible for our observations. Although we confirmed that CFU-Hill was reduced in the peripheral blood of irradiated mice (data not shown), our method could not distinguish those cells from the leukocyte population. Second, irradiation is not a physiological condition, and this must be kept in mind when interpreting the implications of our results for actual disease states.

\section{Conclusion}

In conclusion, our results suggest that BM-derived cells are differentially involved in physiological angiogenesis and pathological angiogenesis in the mouse retina. This raises the possibility that pathological angiogenesis, a key change in many ischemic, vision-threatening retinal diseases, increases when BM-derived cells lose their function. Although further studies are needed to confirm this finding, our results provide new insights into the pathology of retinal neovascular diseases.

\section{Acknowledgements}

The authors appreciate the technical advice and support of colleagues Manabu Sasahara, Atsuko Urasaki, and Junko Nakamura. The present study was supported by the Ministry of Education, Science, and Culture of Japan (Grant \#17689045). 


\section{References}

[1] Jonathan S, Tailoi CL, Jacob P, et al.Roles of Vascular Endothelial Growth Factor and Astrocyte Degeneration in the Genesis of Retinopathy of Prematurity. Invest Ophthalmol Vis Sci. 37 (1996) 290-299.

[2] Harold F. D. Vascular Permeability Factor/Vascular Endothelial Growth Factor: A Critical Cytokine in Tumor Angiogenesis and a Potential Target for Diagnosis and Therapy. J Clin Oncol. 20 (2002) 94368-94380.

[3] Sata M, Saiura A, Kunisato A, et al. Hematopoietic stem cells differentiate into vascular cells that participate in the pathogenesis of atherosclerosis. Nat Med. 8 (2002) 403-409.

[4] Rajantie I, Ilmonen M, Alminaite A, Ozerdem U, Alitalo K, Salven P. Adult bone marrow-derived cells recruited during angiogenesis comprise precursors for periendothelial vascular mural cells. Blood. 104 (2004) 2084-2086.

[5] Ozerdem U, Alitalo K, Salven P, Li A. Contribution of bone marrow-derived pericyte precursor cells to corneal vasculogenesis. Invest Ophthalmol Vis Sci. 46 (2005) 3502-3506.

[6] Espinosa-Heidmann DG, Reinoso MA, Pina Y, Csaky KG, Caicedo A, Cousins SW. Quantitative enumeration of vascular smooth muscle cells and endothelial cells derived from bone marrow precursors in experimental choroidal neovascularization. Exp Eye Res. 80 (2005) 369-378.

[7] Shirakawa K, Furuhata S, Watanabe I, et al. Induction of vasculogenesis in breast cancer models. Br J Cancer. 87 (2002) 1454-1461.

[8] Asahara T. Endothelial progenitor cells for neovascularization. Ernst Schering Res Found Workshop. (2003) 211-216.

[9] Ziegelhoeffer T, Fernandez B, Kostin S, et al. Bone marrow-derived cells do not incorporate into the adult growing vasculature. Circ Res. 94 (2004) 230-238.

[10] Kinnaird T, Stabile E, Burnett MS, et al. Marrow-derived stromal cells express genes encoding a broad spectrum of arteriogenic cytokines and promote in vitro and in vivo arteriogenesis through paracrine mechanisms. Circ Res. 94 (2004) 678-685. 
[11] Ferrara N. Role of vascular endothelial growth factor in physiologic and pathologic angiogenesis: therapeutic implications. Semin Oncol. 29 (2002) 10-14.

[12] Gardiner TA, Gibson DS, de Gooyer TE, de la Cruz VF, McDonald DM, Stitt AW. Inhibition of tumor necrosis factor-alpha improves physiological angiogenesis and reduces pathological neovascularization in ischemic retinopathy. Am J Pathol. 166 (2005) 637-644.

[13] Smith LE, Wesolowski E, McLellan A, et al. Oxygen-induced retinopathy in the mouse. Invest Ophthalmol Vis Sci. 35 (1994) 101-111.

[14] Otani A, Kinder K, Ewalt K, Otero FJ, Schimmel P, Friedlander M. Bone marrowderived stem cells target retinal astrocytes and can promote or inhibit retinal angiogenesis. Nat Med. 8 (2002) 1004-1010.

[15] Banin E, Dorrell MI, Aguilar E, et al. T2-TrpRS inhibits preretinal neovascularization and enhances physiological vascular regrowth in OIR as assessed by a new method of quantification. Invest Ophthalmol Vis Sci. 47 (2006) 2125-2134.

[16] Asahara T, Murohara T, Sullivan A, et al. Isolation of putative progenitor endothelial cells for angiogenesis. Science 275 (1997) 964-967.

[17] Lyden D, Hattori K, Dias S, et al. Impaired recruitment of bone-marrow-derived endothelial and hematopoietic precursor cells blocks tumor angiogenesis and growth. Nat Med. 7 (2001) 1194-1201.

[18] Kocher AA, Schuster MD, Szabolcs MJ, et al. Neovascularization of ischemic myocardium by human bone-marrow-derived angioblasts prevents cardiomyocyte apoptosis, reduces remodeling and improves cardiac function. Nat Med. 7 (2001) 430436.

[19] Asahara T, Masuda H, Takahashi T, et al. Bone marrow origin of endothelial progenitor cells responsible for postnatal vasculogenesis in physiological and pathological neovascularization. Circ Res. 85 (1999) 221-228.

[20] Chan-Ling T, Baxter L, Afzal A, et al. Hematopoietic stem cells provide repair functions after laser-induced Bruch's membrane rupture model of choroidal neovascularization. Am J Pathol. 168 (2006) 1031-1044. 
[21] Lenk K, Adams V, Lurz P, et al. Therapeutical potential of blood-derived progenitor cells in patients with peripheral arterial occlusive disease and critical limb ischaemia. Eur Heart J. 26 (2005) 1903-1909.

[22] Yamamoto K, Kondo T, Suzuki S, et al. Molecular evaluation of endothelial progenitor cells in patients with ischemic limbs: therapeutic effect by stem cell transplantation. Arterioscler Thromb Vasc Biol. 24 (2004) e192-196.

[23] Erbs S, Linke A, Adams V, et al. Transplantation of blood-derived progenitor cells after recanalization of chronic coronary artery occlusion: first randomized and placebocontrolled study. Circ Res. 97 (2005) 756-762.

[24] Ritter MR, Banin E, Moreno SK, Aguilar E, Dorrell MI, Friedlander M. Myeloid progenitors differentiate into microglia and promote vascular repair in a model of ischemic retinopathy. J Clin Invest. 116 (2006) 3266-3276.

[25] Ishida S, Yamashiro K, Usui T, et al. Leukocytes mediate retinal vascular remodeling during development and vaso-obliteration in disease. Nat Med. 9 (2003) 781-788.

[26] Hou HY, Wang YS, Xu JF, Wang YC, Liu JP. The dynamic conduct of bone marrow-derived cells in the choroidal neovascularization microenvironment. Curr Eye Res. 31 (2006) 1051-1061.

[27] Yodoi Y, Sasahara M, Kameda T, Yoshimura N, Otani A. Circulating hematopoietic stem cells in patients with neovascular age-related macular degeneration. Invest Ophthalmol Vis Sci. 48 (2007) 464-5472. 


\section{Figure legends}

FIGURE 1. The oxygen-induced retinopathy mouse model used in the study. (A) The time course for experimental animals. P7 mice were exposed to a $75 \%$ oxygen atmosphere for five days, irradiated, and moved to normal (room) air until examination on P17. (B) Lead plates were used to shield none, N, the head, H, or all, A, of each mouse from radiation.

FIGURE 2. Mouse blood cell counts, hemoglobin levels, and body weight. (A) Mice irradiated with the indicated dose of radiation. 2 Gy and 4 Gy doses of irradiation effectively depleted leukocytes without affecting erythrocytes or hemoglobin levels. $(n=3)$ (B) Relative body weight change of irradiated mice to the average weight of P13 control mice on the indicated post-natal day according to lead cover. There were no differences in body weight among the groups. $(n=4)(C)$ Blood cell counts and hemoglobin levels in mice according to lead cover. Control, $C(n=9)$, covering the entire body, A ( $\mathrm{n}=7)$, protected mice from leukocyte depletion, but covering only the head, $\mathrm{H}$ $(n=5)$, and no cover at all, $N(n=5)$, induced leukocyte depletion. WBC: white blood cells; RBC: red blood cells; Hb: hemoglobin. 
FIGURE 3. Oxygen-induced retinopathy. (A) Representative image of a flat-mount of the retina of a P12 control mouse. Note that the central area of the retina is avascular. (B) Representative image of a flat-mount of the retina of a P17 control mouse. The avascular area is decreased in size relative to that seen in a retina from a P12 mouse; physiological and tuft-like pathological neovascularization can also be seen. (C-F) Representative images of neovascularization sites in control (higher magnification). (C and D) Intraretinal physiological angiogenesis and (E and F) extraretinal pathologic angiogenesis is visible. Note the differences in the angiogenesis patterns and in astrocyte involvement. ( $\mathrm{G}$ and $\mathrm{H}$ ) Representative images showing how avascular and neovascular areas were calculated. The yellow shading represents neovascular tufts and white shading represents avascular area. Each area was defined and measured using image processing software. Red: CD31 immunofluorescence. Red signal: Panels A-C and E, collagen IV; panel F, GFAP; panel G and H, CD31. Green signal (all panels): CD31. Magnification, $\mathrm{x} 10$ (A, and B), x40 (C, D, E, and F), x4 (G and H).

FIGURE 4. Representative images of avascular and neovascular areas of retinas from mice in each experimental group. (A) P12 mouse; (B) control (non-irradiated) P17 mouse; (C) completely shielded P17 mouse; (D): head-shielded P17 mouse. Compared 
to retinas from mice in the control or the completely shielded groups, retinas from headshielded P17 mice had larger avascular and neovascular areas. (E) Quantitation of the avascular areas in each group. The head-shielded group $(H, n=10)$ had an average avascular area that was significantly larger $(P<0.05)$ than in the other groups $(\mathrm{Control}$, $C(n=9)$, covering the entire body, $A(n=6))$. (F) Quantitation of the neovascular areas in each group. Neovascularization was not observed at P12. At P17, the head-shielded group, $\mathrm{H}$, again had the largest average neovascular area $(P<0.05)$. Magnification, $\mathrm{x} 10$. 
Click here to download high resolution image

\section{Figure 1}

A
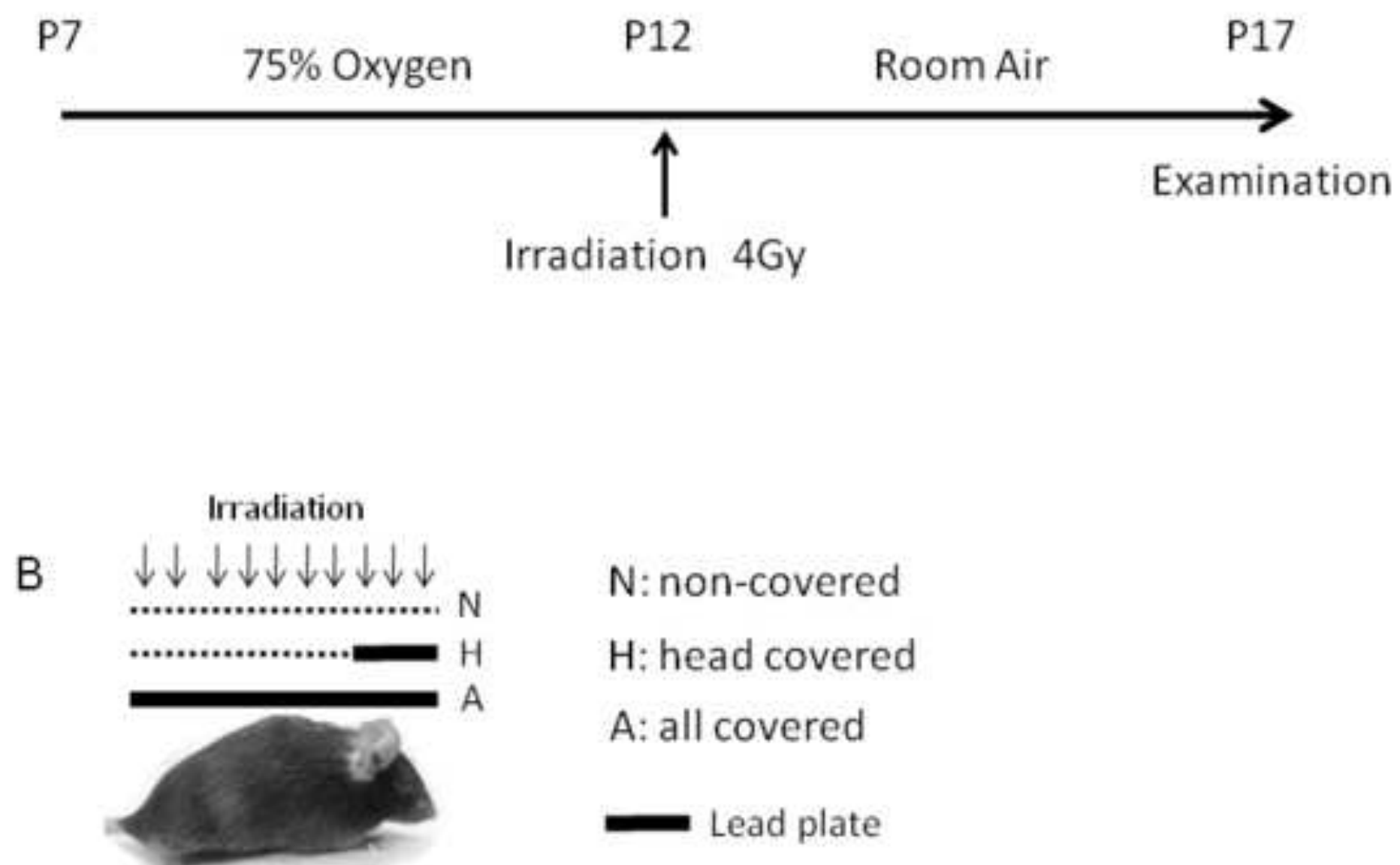


\section{Figure 2}
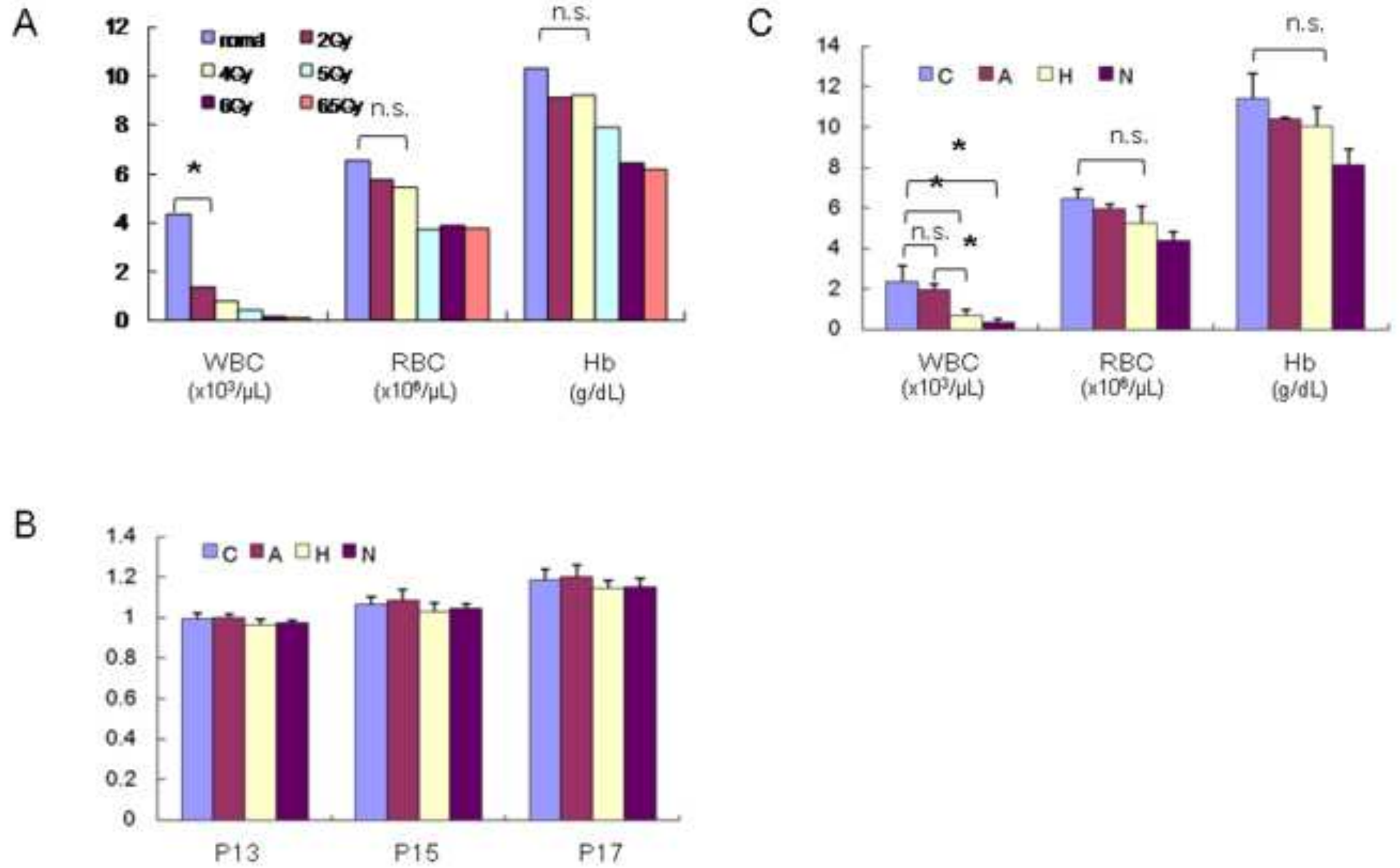
Click here to download high resolution image

\section{Figure 4}
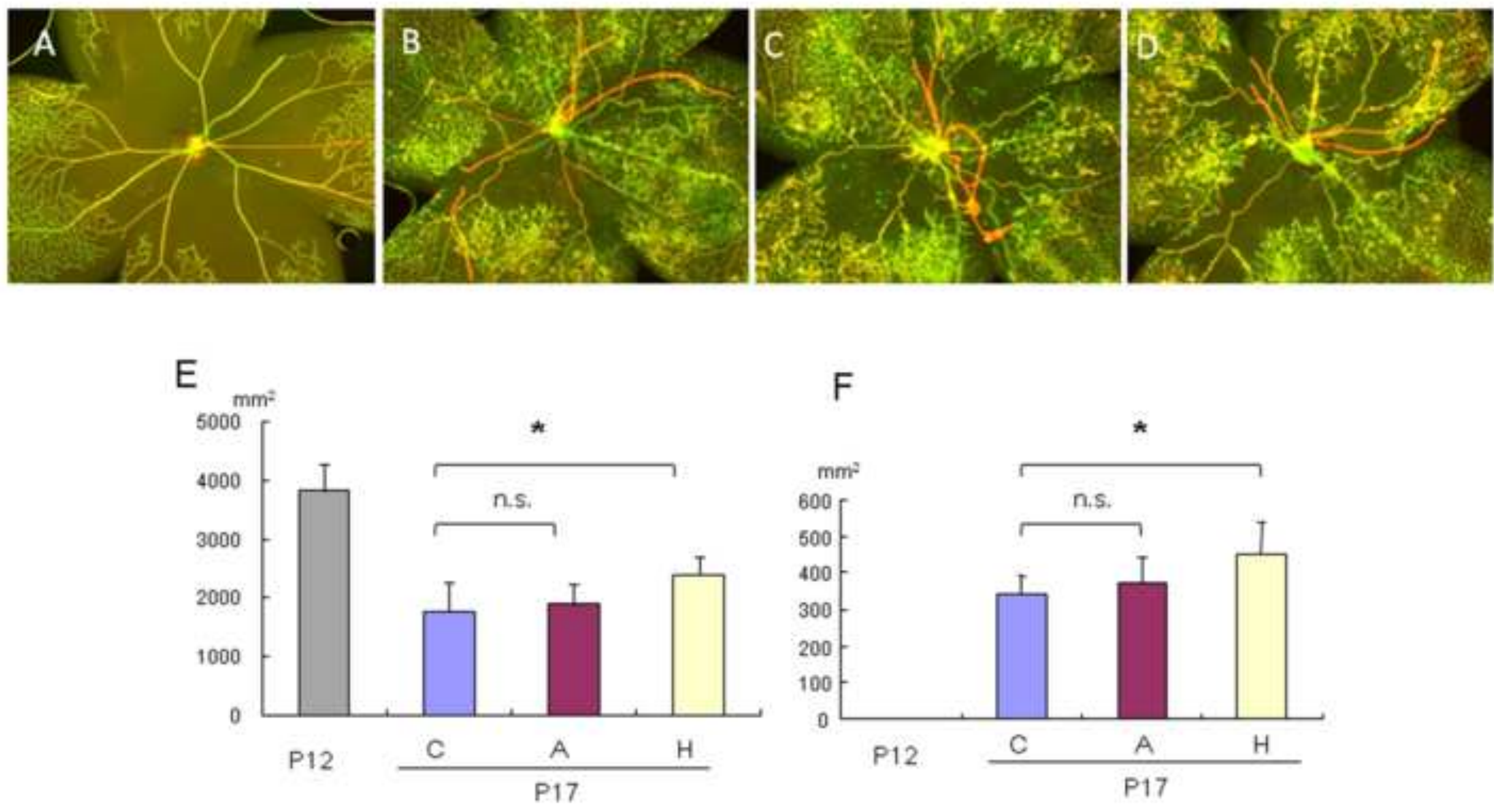
